\title{
Gastrópodes límnicos do Campus de Manguinhos, Fundação Oswaldo Cruz, Rio de Janeiro, RJ
}

\author{
Freshwater snails of the Campus of Manguinhos, \\ Fundação Oswaldo Cruz, Rio de Janeiro, RJ
}

Monica Ammon Fernandez', Silvana Carvalho Thiengo' e Maria Fernanda Boaventura' ${ }^{1}$

\begin{abstract}
Resumo Um levantamento da malacofauna límnina do Campus de Manguinhos, Fundação Oswaldo Cruz, Rio de Janeiro, foi realizado nos últimos dois anos visando comparar as espécies hoje existentes com aquelas encontradas no início deste século. Foram pesquisadas 18 colecões hídricas numa extensão de $880.000 m^{2}$, sendo encontradas em 13 delas as seguintes espécies: Antillorbis nordestensis, Biomphalaria glabrata, Biomphalaria straminea, Lymnaea columella, Melanoides tuberculatus, Physa cubensis, Pomacea glauca $e$ Pomacea lineata. Destacam-se o desaparecimento de Biomphalaria tenagophila, registrada por Lutz em 1918, a introdução de B. straminea e da espécie asiática M. tuberculatus. Nenhum molusco apresentou infecção por Schistosoma mansoni.
\end{abstract}

Palavras-chaves: Gastrópodes límnicos. Vetores. Fundação Oswaldo Cruz. Rio de Janeiro.

\begin{abstract}
A survey of freshwater gastropods of the Campus of Manguinhos, Fundação Oswaldo Cruz, Rio de Janeiro, was carried out during the last two years aiming to compare the current species with those found at the beginning of this century. Among 18 breeding sites in $880,000 \mathrm{~m}^{2}$ of the surveyed area, 13 showed the following species: Antillorbis nordestensis; Biomphalaria glabrata; Biomphalaria straminea; Lymnaea columella; Melanoides tuberculatus; Physa cubensis; Pomacea glauca and Pomacea lineata. Notably, Biomphalaria tenagophila reported by Lutz in 1918, had disappeared and B. straminea and the Asiatic thiarid M. tuberculatus had been introduced. No specimens infected with Schistosoma mansoni were found.
\end{abstract}

Key-words: Freshwater snails. Vectors. Fundação Oswaldo Cruz. Rio de Janeiro.

O primeiro relato da malacofauna límnica em Manguinhos veio com o trabalho de Lutz ${ }^{4}$, que em 1918 registrou a coexistência de Planorbis confusus Lutz $1918 \mathrm{e}$ Planorbis nigricans Spix, 1827, a inexistência de Planorbis olivaceus Spix Wagner, 1827 e a presença de Planorbis melleus Lutz, 1918. Segundo Paraense ${ }^{10}$ e Paraense \& Deslandes $^{12}$, $P$. confusus e $P$. nigricans são sinônimos de Biomphalaria tenagophila (Orbigny, 1835), P. olivaceus de Biomphalaria glabrata (Say, 1818) e P. melleus de Drepanotrema lucidum (Pfeiffer, 1839). A presença de B. glabrata na chácara de Manguinhos foi primeiramente registrada por Jansen², em 1944.

Em 1949, Paraense ${ }^{8}$ coletou exemplares de B. glabrata num córrego dentro dos terrenos do Instituto Oswaldo Cruz e detectou infecção natural por Schistosoma mansoni Sambon, 1907 nestes moluscos ( $8,2 \%)$. Suas investigações revelaram que a contaminação deste criadouro havia sido feita pelas fezes de operários que trabalhavam nas obras de construção de novas dependências do Instituito.
Em 1964, Magalhães ${ }^{5}$ encontrou populações de B. glabrata e B. tenagophila em simpatria em alguns criadouros, bem como exemplares de B. glabrata infectados por $S$. mansoni, cujos índices de infecção chegavam a $2 \%$. Dois anos depois ${ }^{6}$, relatou a ocorrência de um processo de competição biológica nestes criadouros e registrou o deslocamento de B. tenagophila por B. glabrata. Assinalou ainda a presença de exemplares das famílias Ampullariidae, Ancylidae, Physidae e Planorbidae. Em 1983, Jurberg et $\mathrm{al}^{3}$ observaram exemplares de $B$. glabrata em uma vala existente no Campus de Manguinhos e, baseados em estudos comportamentais, caracterizaram fatores que contribuem para a dispersão dos moluscos e repovoamento dos criadouros. Desde então nenhum outro levantamento malacológico em Manguinhos foi publicado. Neste trabalho é feita uma comparação da malacofauna límnica atual do campus de Manguinhos com os dados registrados nos últimos 80 anos.

\footnotetext{
1. Departamento de Malacologia, Instituto Oswaldo Cruz, Fundação Oswaldo Cruz, Rio de Janeiro, RJ.

Endereço para correspondência: Dra. Monica A. Fernandez. Depto. de Malacologia/FIOCRUZ. Av. Brasil 4365, 21045-900, Rio de Janeiro, RJ.

Telefax: 5521 280-5840

e-mail:ammon@ioc.fiocruz.br

Recebido para publicação em 13/3/2000.
} 


\section{MATERIAL E MÉTODOS}

As coletas foram realizadas em 1998 e 1999 em lagos artificiais, valas de drenagem, alagados e nas margens dos rios Jacaré e Faria-Timbó. Os moluscos foram coletados com o auxílio de concha de captura e pinças, postos em frascos plásticos identificados e levados ao laboratório, onde foram acondicionados em aquários previamente preparados contendo água desclorada e filtrada e uma fina camada de argila acrescida de carbonato de cálcio e farinha de ostra.
No laboratório, os moluscos eram examinados individualmente em estereomicroscópio após a exposição à luz de lâmpadas incandescentes (60W), durante 6 horas, para detectar infecção por S. mansoni. Alguns exemplares foram anestesiados, mortos por aquecimento em água a $70^{\circ} \mathrm{C}$ e fixados em solução de Railliet-Henry para a identificação específica e o restante foi examinado após esmagamento entre lâminas de vidro.

\section{RESULTADOS}

Foram pesquisadas 18 coleções hídricas numa extensão de $880.000 \mathrm{~m}^{2}$ (Figura 1), dentre as quais 13 possuíam moluscos límnicos: Antillorbis nordestensis (Lucena 1954); B. glabrata; Biomphalaria straminea

\footnotetext{
1. Casa de Oswaldo Cruz

2. Creche Bertha Lutz

3. ENSP

4. Expansão do Campus

5. Garagem

6. INCQS

7. Pav. Adolpho Lutz

8. Pav. Arthur Neiva

9. Pav. Cardoso Fontes

10. Pav. Carlos A. da Silva

11. Pav. Gomes de Faria

12. Pav. Lauro Travassos

13. Portaria Av. Brasil
}

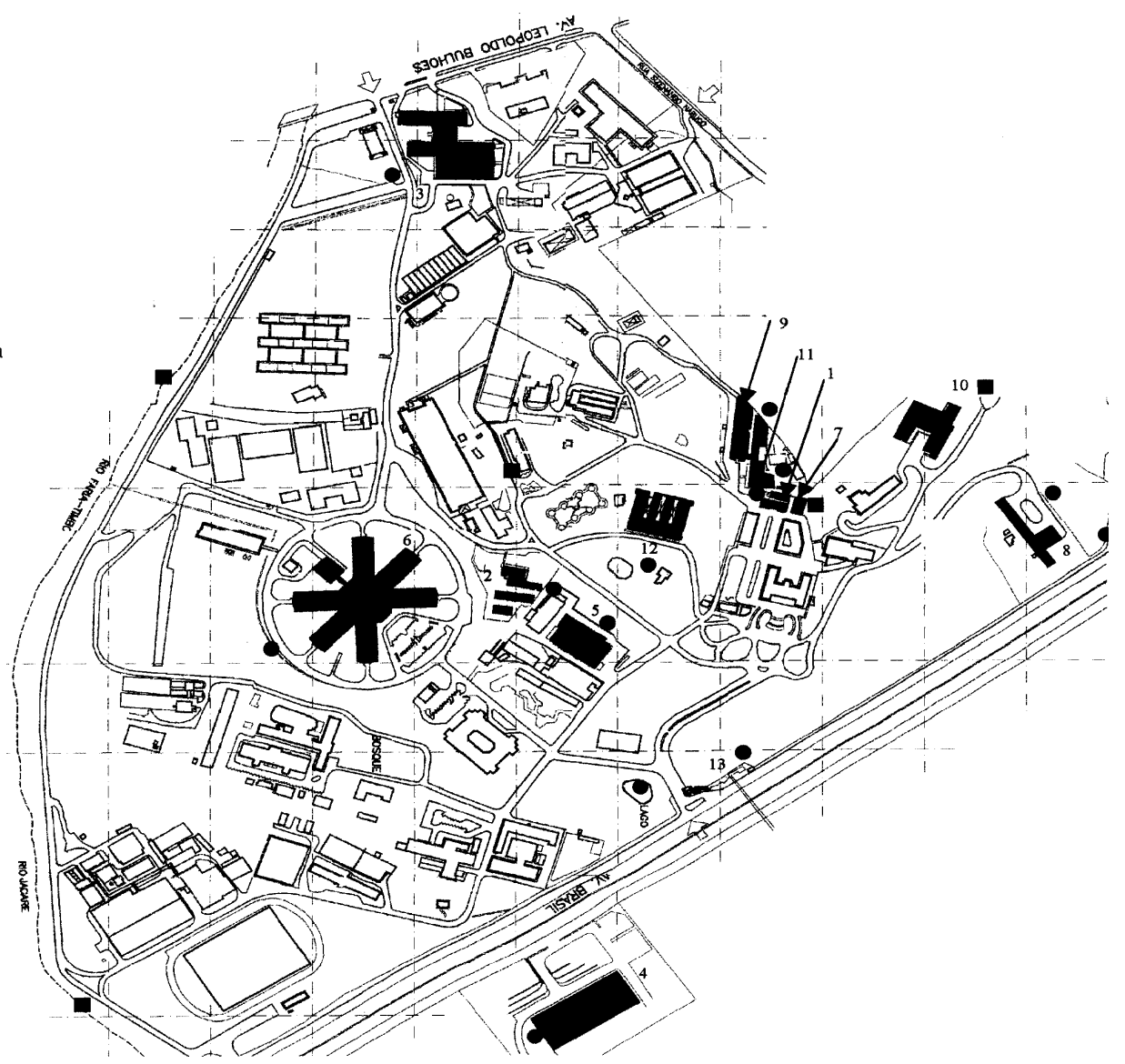

(-) ausência de moluscos

(•) presença de moluscos

Figura 1 - Coleções hídricas pesquisadas no Campus de Manguinhos, Fundação Oswaldo Cruz.

(Dunker, 1848); Lymnaea columella (Say, 1817) Melanoides tuberculatus (Müller, 1774); Physa cubensis Pfeiffer, 1839;
Pomacea glauca (Linné, 1758) e Pomacea lineata (Spix, 1827). Nenhum molusco apresentou infecção por $S$. mansoni. 
Nos rios Faria-Timbó e Jacaré, não foram encontrados moluscos provavelmente por estarem muito poluídos.

A espécie mais freqüente foi $P$. cubensis, presente em 10 dos biótopos, seguida por L. columella (Tabela 1).
B. glabrata e $B$. straminea foram encontradas em três biótopos cada uma, cohabitando com exemplares de fisídeos e limneídeos.

Tabela 1 - Resultado do levantamento malacológico realizado no Campus de Manguinhos, de 1998 a 1999.

\begin{tabular}{|c|c|c|c|c|c|c|c|c|}
\hline Construções & & & & Malacofauna & & & & \\
\hline & $\begin{array}{l}\text { Antillorbis } \\
\text { nordestensis }\end{array}$ & $\begin{array}{c}\text { Biomphalaria } \\
\text { glabrata }\end{array}$ & $\begin{array}{c}\text { Biomphalaria } \\
\text { straminea }\end{array}$ & $\begin{array}{c}\text { Lymnaea } \\
\text { columella }\end{array}$ & $\begin{array}{l}\text { Melanoides } \\
\text { tuberculatus }\end{array}$ & $\begin{array}{l}\text { Physa } \\
\text { cubensis }\end{array}$ & $\begin{array}{c}\text { Pomacea } \\
\text { glaucal }\end{array}$ & $\begin{array}{c}\text { Pomacea } \\
\text { ineata }\end{array}$ \\
\hline Casa de Oswaldo Cruz & & & & & & $\square$ & & \\
\hline Creche Bertha Lutz & & & * & * & & * & & \\
\hline Escola Nacional de & & & & & & $*$ & & \\
\hline Saúde Publica (ENSP) & & & & & & & & \\
\hline Expansão do Campus & & & & & & & & \\
\hline Garagem & & & * & * & & * & & \\
\hline Instituto Nac. Controle & & * & & & * & 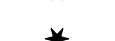 & & * \\
\hline de Qualidade (INCQS) & & & & & $\star$ & & & \\
\hline Pavilhão Arthur Neiva & & * & & & & * & & * \\
\hline Pavilhão Cardoso Fontes & & & & & & & & \\
\hline Pavilhão Gomes de Faria & & $\square$ & & & & & & \\
\hline Pavilhão Lauro Travassos & & & & $\bullet$ & & 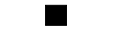 & $\bullet$ & \\
\hline Portaria Av. Brasil & & & $\square \bullet$ & • & & $\square$ & $\bullet$ & \\
\hline
\end{tabular}

\section{DISCUSSÃO}

As únicas espécies encontradas por Lutz (1918), $B$. tenagophila e $D$. lucidum, não foram observadas. É provável que $B$. tenagophila tenha desaparecido entre as décadas de 60 e 70, uma vez que Paraense ${ }^{9}$ ao listar as localidades brasileiras que apresentavam planorbídeos vetores da esquistossomose, cita para Manguinhos apenas $B$. glabrata. Com relação a $D$. lucidum, o desaparecimento pode ter ocorrido antes de 1966 já que Magalhães ${ }^{6}$, em seu levantamento malacológico, não cita esta espécie.

A introdução de Biomphalaria glabrata, espécie atualmente encontrada em três biótopos, ocorreu em 1917 quando Lutz depositou alguns exemplares, provenientes de Aracajú, num tanque aberto ao ar livre, junto ao seu laboratório ${ }^{5}$. Biomphalaria glabrata tem seu maior domínio no Sudeste da Bahia e na metade oriental de Minas Gerais somada ao território adjacente do Espírito Santo, estende-se continuamente em todas as direções incluindo a faixa costeira e interiores em Sergipe, Alagoas, Pernambuco, Paraíba e Rio Grande do Norte. Ocorrências isoladas são citadas para o Pará, Maranhão, Bahia, Goiás, Rio de Janeiro, São Paulo, Paraná e recentemente, Rio Grande do Sul ${ }^{1}{ }^{11}$.

Até 1986, a ocorrência no Estado do Rio de Janeiro de $B$. straminea, espécie vetora do $S$. mansoni mais amplamente distribuída no país, restringia-se a dois municípios, Duque de Caxias e Paracambi ${ }^{11}$. Com o levantamento malacológico do estado que vem sendo realizado desde 1995 pelos autores ${ }^{16}{ }^{17}$, a distribuição desta espécie foi ampliada: Guapimirim, Itaboraí, Magé,
Mangaratiba, Maricá, Miguel Pereira, Niterói, Nova Iguaçu, Petrópolis, São Gonçalo e Vassouras. A inexistência de levantamentos malacológicos anteriores não nos permite afirmar que essa espécie esteja em expansão, como tem sido observado em países do Hemisfério Leste ${ }^{71419}$, onde sua introdução foi atribuída a pássaros de hábitos aquáticos e ao incremento do comércio de plantas para aquariofilistas.

Magalhães ${ }^{5}$ considerou o encontro de A. nordestensis e Drepanotrema cimex (Moricand, 1839) como inéditos, porém na Coleção Malacológica do Instituto Oswaldo Cruz existem amostras registradas anteriormente: $D$. cimex em 1956 (CMIOC $\mathrm{n}^{\circ}$ 272) e 1962 (CMIOC $\mathrm{n}^{\circ}$ 632), e A. nordestensis em 1963 (CMIOC n० 824). No levantamento atual somente $A$. nordestensis foi encontrado.

Foram observadas duas espécies de ampulariídeos: P. glauca, que se distribui pelo Caribe e norte do Brasil, e P. lineata, com distribuição principalmente litorânea, desde o nordeste brasileiro até São Paulo. Na Coleção Malacológica do IOC o primeiro registro de $P$. lineata (CMIOC $n^{\circ} 2737$ ) é de março de 1982, com exemplares coletados em valas de drenagem, onde ocorrem até hoje, e de abril do mesmo ano para $P$. glauca (CMIOC $n^{\circ} 2338$ ), espécimes coletados em um tanque, extinto atualmente, atrás do prédio onde hoje funciona a Casa de Oswaldo Cruz e em lagos artificiais na entrada do Campus pela Avenida Brasil e próximo ao Pavilhão Lauro Travassos. 
Quanto aos Physidae, os primeiros registros são de 1962, com espécimes de $P$. cubensis (CMIOC n 631), única espécie presente até hoje no campus, e de Physa marmorata Guilding, 1828 (CMIOC n 633 ). Com relação a L. columella, o registro mais antigo data de 1975 (CMIOC $n^{\circ}$ 2132). Nenhum representante da família Ancylidae foi encontrado no presente levantamento.

A expansão do tiarídeo asiático $M$. tuberculatus para vários países de regiões tropicais tem sido largamente citada na literatura, principalmente no Caribe, devido a sua utilização em programas de controle biológico dos vetores do $S$. mansoni $i^{13} 14$. No Brasil, onde foi citada pela primeira vez em São Paulo por Vaz et $a^{18}$, populações dessa espécie, muitas vezes extremamente densas, têm sido observadas em vários municípios do Estado do Rio de Janeiro ${ }^{16}$. Com relação ao Campus de Manguinhos, na vala próxima ao prédio do INCQS, único criadouro em que ocorre, foi observado que as populações previamente existentes de $B$. glabrata e $P$. lineata, foram sendo deslocadas e reduzidas a um trecho muito curto da vala, onde atualmente pequenas populações cohabitam com milhares de exemplares de $M$. tuberculatus que ocupam também todo o restante do criadouro.

As áreas pesquisadas diferiram bastante daquelas existentes anteriormente, pois a maioria dos criadouros naturais, várzeas e brejos, encontram-se extintos atualmente, devido ao processo de urbanização ocorrido no campus e nas áreas circunvizinhas. As mudanças observadas na malacofauna límnica provavelmente decorreram tanto da ação antrópica quanto do processo de competição biológica entre as espécies.

\section{AGRADECIMENTOS}

Os autores dedicam este trabalho ao Instituto Oswaldo Cruz, em homenagem ao centenário de sua fundação, 25 de Maio de 1900.

\section{REFERÊNCIAS BIBLIOGRÁFICAS}

1. Carvalho OS, Nunes IM, Caldeira RL. First report of Biomphalaria glabrata in the State of Rio Grande do Sul, Brazil. Memórias do Instituto Oswaldo Cruz 93: 39-40, 1998.

2. Jansen G. Sobre a validade do Australorbis centimetralis (Lutz, 1918). (Nota prévia). Memórias do Instituto Oswaldo Cruz 40: 201-208, 1944.

3. Jurberg P, Coelho da Silva CLPA, Barreto MGM, Soares MS. Rheotaxis of Biomphalaria glabrata on vertical substrates and its role in the recolonization of habitats treated with molluscicides. Memórias do Instituto Oswaldo Cruz 83: 165-174, 1988.

4. Lutz A. Caramujos de água doce do gênero Planorbis, observados no Brasil. Memórias do Instituto Oswaldo Cruz 10: 65-82, 1918.

5. Magalhães LA. Moluscos planorbídeos do Estado da Guanabara. Revista Brasileira de Biologia 24: 277-288, 1964.

6. Magalhães LA. Estudo de uma população de Biomphalaria glabrata (Say, 1818), recentemente introduzida no Estado da Guanabara. Folia Clinica et Biologica 35: 102-120, 1966.

7. Meier-Brook C. A snail intermediate host of Schistosoma mansoni introduced into Hong Kong. Bulletin of the World Health Organization 51: 661, 1974.

8. Paraense WL. Observações adicionais sobre o sexo do "Schistosoma mansoni" nas infestações por cercárias de um único molusco. Memórias do Instituto Oswaldo Cruz 47: 535546, 1949

9. Paraense WL. Planorbídeos hospedeiros intermediários do Schistosoma mansoni. In: Cunha AS (ed) Esquistossomose mansoni, Universidade de São Paulo, São Paulo, p. 13-30, 1970.

10. Paraense WL. Estado atual da sistemática dos planorbídeos brasileiros. Arquivos do Museu Nacional, RJ, 55: 105-128, 1975.

11. Paraense WL. Distribuição dos caramujos no Brasil. In: Reis FA, Faria I, Katz N (eds) Modernos conhecimentos sobre esquistossomose mansônica, Biblioteca Academia Mineira de Medicina 14: 117-128, 1986.
12. Paraense WL, Deslandes N. Observations on the morphology of Australorbis nigricans. Memórias do Instituto Oswaldo Cruz 53: 121-134, 1955.

13. Pointier JP. The introduction of Melanoides tuberculata (Mollusca: Thiaridae) to the island of Saint Lucia (West Indies) and its role in the decline of Biomphalaria glabrata, the snail intermediate host of Schistosoma mansoni. Acta Tropica 54: 13-18, 1993.

14. Pointier JP, Guyard A, Mosser A. Biological control of Biomphalaria glabrata and $B$. straminea by the competitor snail hiara tuberculata in a transmission site of schistosomiasis in Martinique, French West Indies. Annals of Tropical Medicine and Parasitology 83: 263-269, 1989.

15. Prentice MA. Displacement of Biomphalaria glabrata by the snail hiara granifera in field habitats in St Lucia, West Indies. Annals of Tropical Medicine and Parasitology 77: 51-59, 1983.

16. Thiengo SC, Fernandez MA, Boaventura MF, Stortti MA. A survey of freshwater gastropods in the Microrregião Serrana of State of Rio de Janeiro, Brazil. Memórias do Instituto Oswaldo Cruz 93 (supl): 233-234, 1998.

17. Thiengo SC, Fernandez MA, Boaventura MF, Stortti MA, Araujo FP. A survey of freshwater gastropods in the Mesorregião Metropolitana of the State of Rio de Janeiro, Brazil. In: Anais do VII Simpósio Internacional de Esquistossomose, p. 166, 1999.

18. Vaz JF, Teles HMS, Correa MA, Leite SPS. Ocorrência no Brasil de Thiara (Melanoides) tuberculata (O.F. Muller, 1774) (Gastropoda, Prosobranchia), primeiro hospedeiro intermediário de Clonorchis sinensis (Cobbold, 1875) (Trematoda, Platyhelmintes). Revista de Saúde Pública 20: 318-322, 1986.

19. Yipp MW. Distribution of the schistosome vector snail, Biomphalaria straminea (Pulmanata: Planorbidae) in Hong Kong. Journal of Molluscan Studies 56: 47-55, 1990. 